Review Article

\title{
An Overview of Systematic Reviews of Randomized Controlled Trials on Acupuncture Treating Migraine
}

\author{
Xia-tian Zhang $\mathbb{D D}^{1}{ }^{1}$ Xin-yi Li, ${ }^{1}$ Chen Zhao ${ }^{D},{ }^{2}$ Ye-yin Hu, ${ }^{1}$ Yi-yi Lin, ${ }^{1}$ He-qing Chen, ${ }^{1}$ \\ Zhao-feng Shi, ${ }^{1}$ Xiao-yu Zhang, ${ }^{1}$ Hong-cai Shang, ${ }^{1}$ and Gui-hua Tian $\left(^{1}{ }^{1}\right.$ \\ ${ }^{1}$ Key Laboratory of Chinese Internal Medicine of MOE and Beijing, Dongzhimen Hospital, \\ Beijing University of Chinese Medicine, Beijing 100700, China \\ ${ }^{2}$ Institute of Basic Research in Clinical Medicine, China Academy of Chinese Medical Sciences, Beijing 100700, China \\ Correspondence should be addressed to Gui-hua Tian; rosetgh@163.com
}

Received 30 March 2019; Revised 12 June 2019; Accepted 1 July 2019; Published 29 October 2019

Guest Editor: Fang Zeng

Copyright ( $\odot 2019$ Xia-tian Zhang et al. This is an open access article distributed under the Creative Commons Attribution License, which permits unrestricted use, distribution, and reproduction in any medium, provided the original work is properly cited.

Objectives. To review the evidence of acupuncture for acute and preventive treatment of migraine for further awareness of the effect of acupuncture for migraine. Design. An overview of systematic reviews and meta-analyses (SR/MAs) for randomized controlled trials. Material and Methods. We searched PubMed, Embase, the Cochrane Library, China Knowledge Resource Integrated Database, VIP Chinese Journal Full Text Database, WANFANG Data, and China Biology Medicine disc from their establishment to May 27, 2018. SR/MAs of randomized controlled trials comparing the effect of the acupuncture intervention with another treatment control in migraine patients were included. Results. 428 SRs were identified, and 15 of them were included. Only 4 SR/MAs were assessed by GRADE, which showed certainty of most evidence being low or very low. Assessed by AMSTAR2 , fourteen was critically low rating overall confidence in the results, and 1 was low rating overall confidence in the results. Evidence suggested that acupuncture has a significant advantage of pain improvement, efficacy, and safety relative to blank control, sham acupuncture, or drug treatment, but some of these results are contradictory. Conclusions. We found that acupuncture on treating migraine has the advantage for pain improvement and safety, but the quality of SR/MAs of acupuncture for migraine remains to be improved.

\section{Introduction}

Migraine is a common disabling primary headache disorder [1]. Epidemiological studies revealed that the global agestandardized prevalence of migraine was $14.4 \%(13.8-15.0 \%)$ in 2016, and this figure was $18.9 \%(18.1-19.7)$ for women and 9.8\% (9.4-10.2) for men; in addition, the prevalence by ages increased significantly until reaching a peak between 35 and 39 years and decreased smoothly after 40 years, and for China, the prevalence of migraine was $9.3 \%(8.5-10.1 \%)$ in 2009 [2, 3]. Migraine has two major subtypes, "migraine without aura" and "migraine with aura," and the mechanism of migraine is related to vascular, pain pathway, central system [1]. Acute treatments for migraine usually include using aspirin or triptans (a highly selective serotonin $5-\mathrm{HT}_{1 \mathrm{~B}}$ and $5-\mathrm{HT}_{1 \mathrm{D}}$ receptor agonists), and other drugs, whereas preventive treatment adopts drugs, cognitive behavioral therapy, and other therapeutic methods $[4,5]$.

Acupuncture is a kind of physical therapy, which is an essential component of "Traditional Chinese Medicine" (TCM) [6]. TCM theory believes that a kind of critical energy called Qi maintains the regular operation of the human body and this energy flows in a network of channels called meridians. The theory of TCM also believes the human body will suffer from illness or disease once this flow is abnormal, which can be recovered by inserting the needle in some specified points on the channels of meridians of the human body like acupuncture. Acupuncture is often used to treat headache including migraine [7], and its therapeutic mechanism may be related to its regulation of nitric oxide synthase and 5-hydroxytryptamine (5-HT) gene ${ }_{1 \mathrm{~F}}$ expression to improve cerebral vasodilation and contraction [8]. 
Systematic reviews can provide a robust review of the effectiveness of clinical interventions [9]; according to the literature search, clinical researchers have completed a number of systematic reviews of acupuncture treating migraine. To summarize the evidence of acupuncture for migraine [10] and show the effect of acupuncture for acute and preventive treatment of migraine, the researchers carried out an overview of systematic reviews/meta-analyses (SR/MA) of randomized controlled trials (RCTs) on acupuncture for migraine.

\section{Material and Methods}

2.1. Studies Searches. We searched PubMed, Excerpta Medica database (Embase), the Cochrane Library, China Knowledge Resource Integrated Database, VIP Chinese Journal Full Text Database, WANFANG Data, and China Biology Medicine disc from their establishment to May 27, 2018, with the following search terms: "migraine*," "status migrainosus," "sick headache," "acupuncture," "needle," "needling," "thorns," "dry-needling," "body-acupuncture," "stitch," "tapping," "electroacupuncture," "electro-acupuncture," "prick," "pricking," "bloodletting," "puncturing collateral," "bleeding therapy," "Acusector," "quick puncture," "blood-letting," "systematic review," "SR," "systematic evaluation," "systematic assessment," "meta-analysis*," and "Cochrane review."

2.2. Inclusion and Exclusion Criteria. The inclusion criteria were as follows: (1) Type of studies: SR/MAs of randomized controlled trials, (2) Type of participants: migraine diagnosed by any internationally recognized or accepted clinical guideline or consensus like The International Classification of Headache Disorders, 3rd edition (beta version) [1], (3) Type of interventions: the experimental groups were treated with acupuncture, which contained acupuncture with electrical stimulation and other acupuncture techniques with needles inserted into the skin, (4) Type of control: the control groups were treated with other blank controls, placebo, drug treatments, or other TCM treatments (5) Type of Outcomes: the primary outcomes were as follows: (a) pain intensity rated by measure tools: visual analogue scales (VAS), numerical rating scales (NRS) score, and verbal rating scale (VRS) score; (b) usage of (rescue) analgesics (any continuous or rank measures available), which was recommended by Initiative on Methods, Measurement, and Pain Assessment in Clinical Trials [11]; and (c) severe events; secondary outcome was frequency of migraine attacks (per 4 weeks or per month), headache frequency or times (per 4 weeks or per month), the number of migraine days (per 4 weeks or per month), the number of headache days (per 4 weeks or per month), and effective rate (curing rate or improving rate evaluated by researchers or subjects of studies included), which is lack of international recognition but commonly used in some Chinese literature. With the following situations, the studies were excluded: (1) non-SR/ MA, (2) non-Chinese and English studies, and (3) no full-text studies were available.
2.3. Data Extraction. All searched studies were imported into Endnote X8.0 for document management. Two coauthors (YH, HC) screened the titles and abstracts of all studies according to the inclusion and exclusion criteria, and then full texts of possible relevant studies were screened. Any disagreement was resolved by consulting another coauthor (XL).

EpiData 3.1 was used to extract the following items data by three coauthors (YL, HC, and XL): (1) Basic information of included SR/MAs: the number of studies, interventions, and main conclusions; (2) Methodology and evidence certainty of included SR/MAs: methodological information of SR/MA that recorded in AMSTAR-2 (a measurement tool to assess systematic reviews) [12], certainty of evidences reviewed by GRADE (Grading of Recommendations, Assessment, Development, and Evaluations) [13]; (3) Effect of intervention: outcomes of SR/MAs.

2.4. Assessment of Methodology of Included Reviews. AMSTART-2 was used to assess the methodological quality of included SR/MAs: When no or only 1 nonkey items did not conform, inferring rating overall confidence in the results of the SR/MA as high; when more than 1 nonkey item did not conform, inferring rating overall confidence in the results of the SR/MA as moderate; when 1 key item did not conform with nonkey items conforming or not conforming, inferring rating overall confidence in the results of the SR/ MA as low; and when more than 1 key items did not conform with nonkey items conforming or not conforming, inferring rating overall confidence in the results of the SR/MA as critically low.

2.5. Statistical Analysis. We use frequency to show data (types of included studies' therapies, types of outcome indexes, AMSTAR-2 compliance items, outcome indexes of meta-analysis, meta-analysis results, and so on). Microsoft Excel 365 was used for data visualization: Reformat data extracted from the underlying systematic reviews in tables.

According to SR/MAs, the results of quantitative synthesis were reported in the form of standard mean deviation (SMD), weighted mean deviation (WMD), odds ratio (OR), or relative risk (RR). And the results were also reported with 95\% confidence intervals (CI).

\section{Results}

3.1. Study Selection Process. A total of 428 studies were initially searched. 52 studies were selected after reading the title and abstract, and 15 SR/MAs were included in the overview after viewing full texts [7, 14-27], which included 2 Cochrane systematic reviews $(9.5 \%)[7,14]$ and 19 nonCochrane system reviews (90.4\%). The study selection process is shown in Figure 1.

3.2. Study Characteristics. The basic information of the included studies is presented in Table 1. For the 15 SR/MAs, 13 studies included migraine patients [14-22, 24-27], 1 


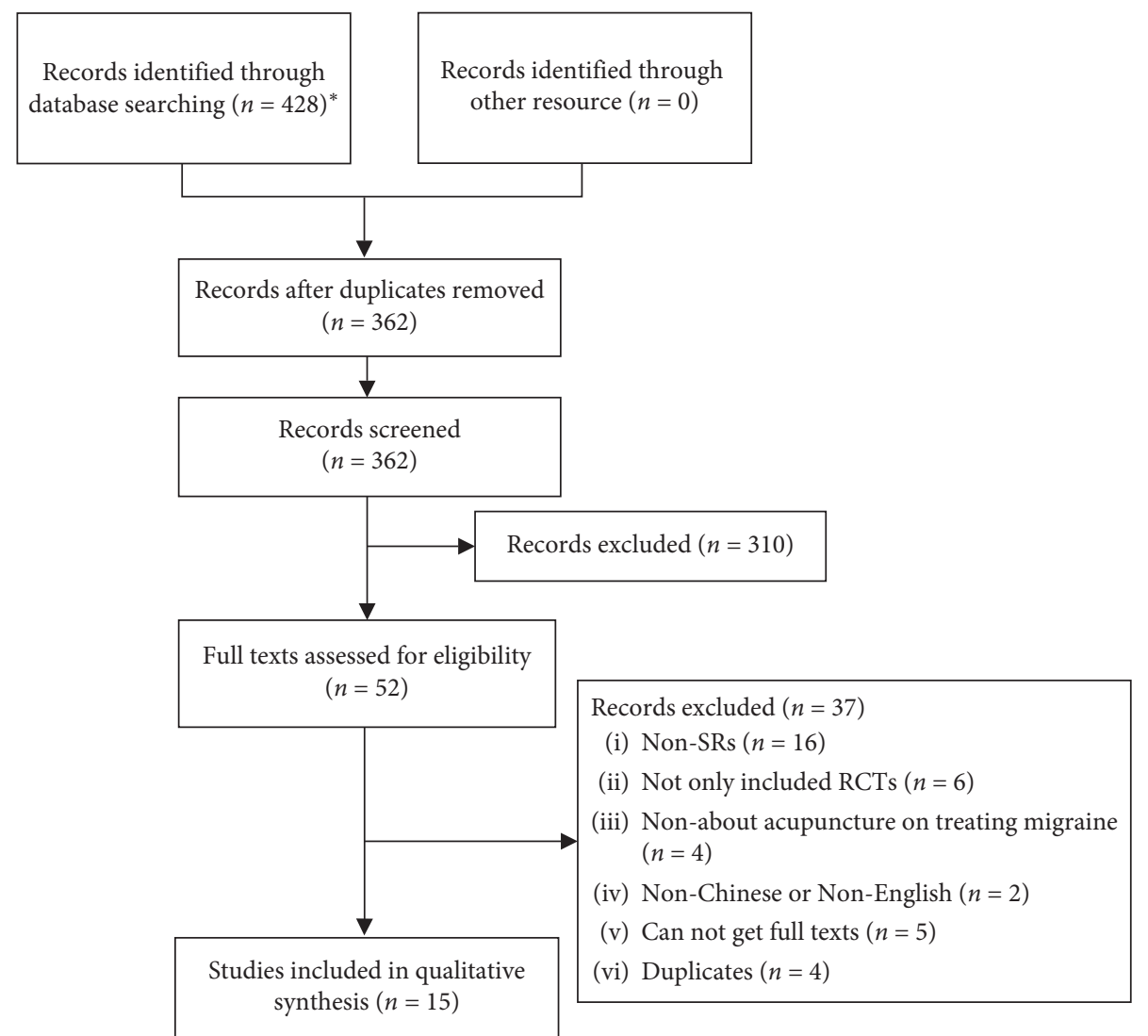

FIGURE 1: Studies selection for acupuncture treating migraine SR/MAs flow diagram.

included episodic migraine patients (participants had been diagnosed with episodic migraine) [7] and one study included menstrual migraine patients (participants had been diagnosed by the International Classification of Headache Disorders s-1/ $2 / 3$ or criteria of menstrual migraine in Criteria for the Diagnosis and Therapeutic Effect of Diseases and Symptoms in Traditional Chinese Medicine [30] published by the State Administration of Traditional Medicine of China) [23]. For the timing of intervention, 11 studies did not define acute or preventive treatment, whereas 1 study was for acute treatment [25] and 3 studies were for preventive treatment [7, 24, 26].

3.3. Certainty of Evidences of Systematic Reviews Included. In the certainty of the evidence, only 4 studies were reviewed by GRADE $[7,16,23,24]$, the certainty of the evidence of these studies was like Table 2.

3.4. Methodological Quality. In the aspect of methodological quality, no studies were all conformed, and one was low rating overall confidence in the results [7], and fourteen were critically low rating overall confidence in the results [14-27], like Table 3.

\subsection{Meta-Analyses Outcomes of Intervention}

3.5.1. Outcomes of Pain Improvement. For the VAS score, 2 SRs reported it as meta-analyses (MAs) outcomes like
Table $4[18,25]$. For the headache situation, 5 SRs reported it as MAs outcomes like Table $5[7,14,20,24,26]$.

3.5.2. Outcomes of Efficacy. For efficacy, 12 SRs reported it as MAs outcome indexes like Table $6[7,14-17,19,20$, 22-24, 26, 27].

3.5.3. Outcomes of Safety. For adverse events, 9 SR/MAs reported adverse events [7, 14-16, 21, 23, 24, 26, 27], but only 4 reported it as MAs outcome indexes like Table $7[7,14,16,24]$.

\section{Conclusions of SR/MAs Included}

In terms of conclusion, the results of all 15 (100\%) SR/MAs were positive. For treatment, 6 SR/MAs reported acupuncture had superiority relative to drugs [7, 16, 17, 19, 26, 27]; 4 SR/ MAs reported acupuncture had superiority relative to sham acupuncture, drugs [14, 18, 22, 24]; 3 SR/MAs reported acupuncture had superiority relative to sham acupuncture [20, 21, 25]; 1 SR/MA reported acupuncture had superiority relative to drugs, other TCM treatments [16]. 1 SR/MA reported that acupuncture had superiority in treating migraine, but did not mention the control group in the conclusions [23].

\section{Discussions}

5.1. Summary of Main Findings. This overview included a considerable number of SR/MAs, illustrating that 


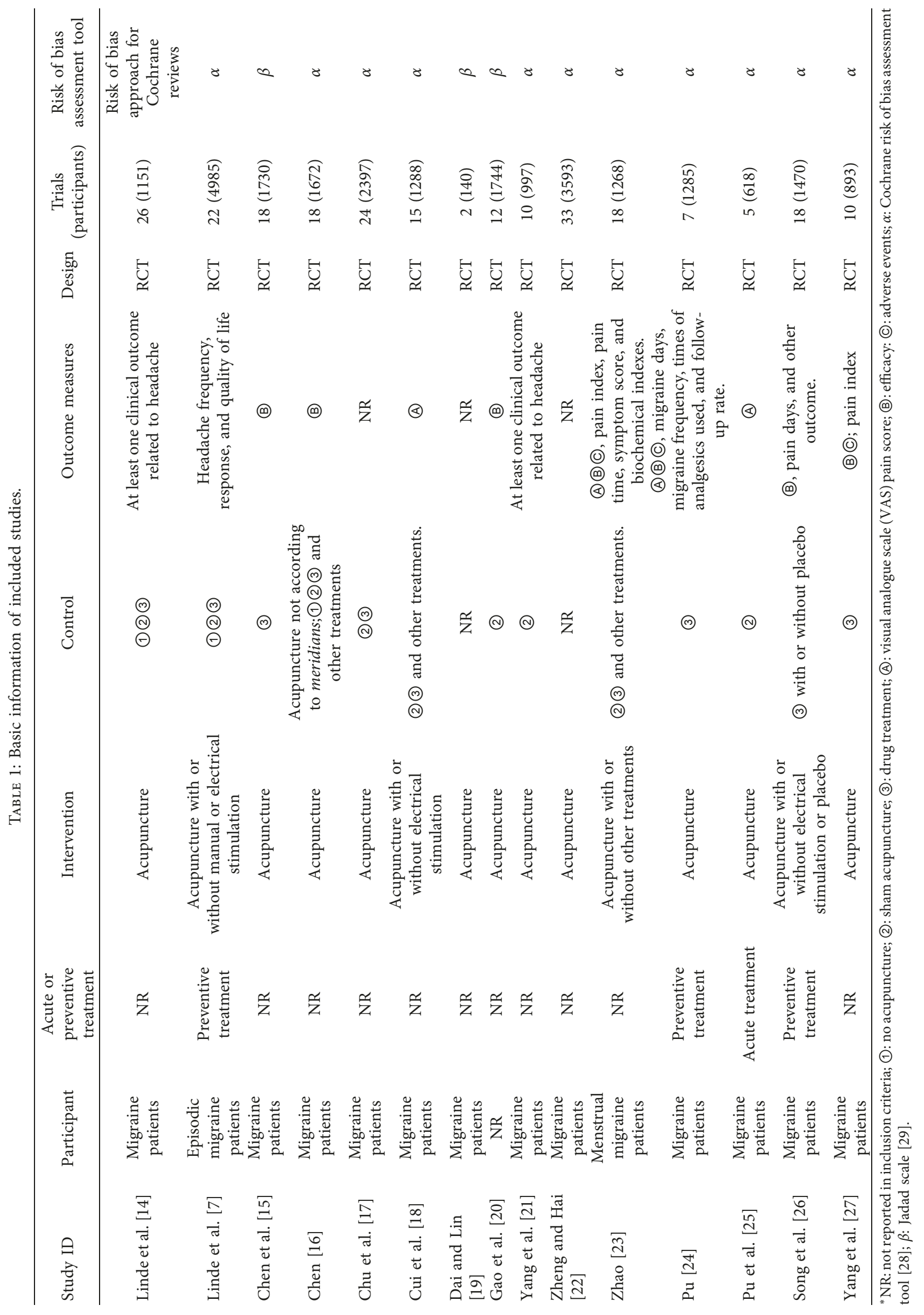


TABLE 2: Certainty of evidences reviewed by GRADE.

\begin{tabular}{llll}
\hline Study ID & Intervention & Control & Outcomes
\end{tabular}

\begin{tabular}{lc}
\hline Acupuncture & $\begin{array}{c}\text { No treatment/usual } \\
\text { care }\end{array}$ \\
Linde et al. & \\
[7] & Sham acupuncture \\
& \\
& \\
& \\
& \\
& \\
& \\
& \\
& \\
&
\end{tabular}

Headache frequency (after treatment) assessed with days per month; response (after treatment and follow-up) assessed with proportion of participants with at least $50 \%$ headache frequency reduction

Headache frequency (follow-up) assessed with days per month

Headache frequency (after treatment and follow-up) assessed with days per month; response (after treatment and follow-up) assessed with proportion of participants with at least $50 \%$ headache frequency reduction

Number of participants dropping out because of adverse effects

Number of participants reporting adverse effects

Headache frequency (after treatment and follow-up) assessed with days per month; response (after treatment and follow-up) assessed with proportion of participants with at least $50 \%$ headache frequency reduction; number of participants dropping out because of adverse effects; number of participants reporting adverse effects

$$
\text { Effective rate }
$$

Low moderate

Headache frequency, headache intensity, length of headache, combined symptoms, and symptom score

Effective rate, headache frequency, and combined symptoms

Other TCM treatment

Chen [16]

Acupuncture

NR

Headache intensity and length of headache

VAS score and adverse events

Acupuncture

TCD-MCA;TCD-ACA; TCD-PCA;TCD$\mathrm{VA}$

TCD-BA

Effective rate, pain index, headache

Acupuncture

Drug treatment

Acupuncture with acupuncture on ear

Drug treatment

Zhao [23] intensity, length of headache, combined symptoms score, and symptom score

Effective rate of mild patients

Moderate

Low

Moderate

Low

High

Moderate

Effective rate, effective rate of moderate patients, effective rate of severe patients, headache score, $\beta$-EP, Vasopressin, NO,

PGF, ET-1, and adverse effects.

Moderate

Low

Very low

Moderate

Low

Very low

Very low

Low

Very low

$$
\begin{gathered}
\text { Acupuncture with TCM drug } \\
\text { treatment } \\
\text { Acupuncture with electrical } \\
\text { stimulation }
\end{gathered}
$$

Acupuncture for bloodletting
Drug treatment

TCM drug treatment
Drug treatment

Effective rate

Effective rate

Effective rate, headache intensity, and length of headache

Migraine days (5-6 months); migraine times

(3-4 months); effective rate (5-6 months)

Migraine days (3-4 months), migraine times

(5-6 months), headache intensity (5-6

$\mathrm{Pu}[24] \quad$ Acupuncture Drug treatment months), and number of use of analgesics (56 months)

Headache intensity (3-4 months), number of use of analgesics (3-4 months), effective rate (3-4 months), adverse effects, and follow-up 
TABLE 3: AMSTAR-2 assessment table.

\begin{tabular}{|c|c|c|c|c|c|c|}
\hline \multirow{2}{*}{ AMSTAR-2 items } & \multicolumn{2}{|c|}{ Yes } & \multicolumn{2}{|c|}{ Partially yes } & \multicolumn{2}{|c|}{ No } \\
\hline & $n$ & $\%$ & $N$ & $\%$ & $n$ & $\%$ \\
\hline A01 & 12 & 80.0 & 0 & 0.0 & 3 & 20.0 \\
\hline A02 & 0 & 0.0 & 0 & 0.0 & 15 & 100.0 \\
\hline A03 & 15 & 100.0 & 0 & 0.0 & 0 & 0.0 \\
\hline A04 & 0 & 0.0 & 14 & 93.3 & 1 & 6.7 \\
\hline A05 & 12 & 80.0 & 0 & 0.0 & 3 & 20.0 \\
\hline A06 & 12 & 80.0 & 0 & 0.0 & 3 & 20.0 \\
\hline $\mathrm{A} 07$ & 2 & 13.3 & 1 & 6.7 & 12 & 80.0 \\
\hline A08 & 4 & 26.7 & 8 & 53.3 & 3 & 20.0 \\
\hline A09 & 12 & 80.0 & 3 & 20.0 & 0 & 0.0 \\
\hline A10 & 0 & 0.0 & 0 & 0.0 & 15 & 100.0 \\
\hline A11 & 15 & 100.0 & 0 & 0.0 & 0 & 0.0 \\
\hline A12 & 9 & 60.0 & 0 & 0.0 & 6 & 40.0 \\
\hline A13 & 10 & 66.7 & 0 & 0.0 & 5 & 33.3 \\
\hline A14 & 9 & 60.0 & 0 & 0.0 & 6 & 40.0 \\
\hline A15 & 9 & 60.0 & 0 & 0.0 & 6 & 40.0 \\
\hline A16 & 1 & 6.7 & 0 & 0.0 & 14 & 93.3 \\
\hline
\end{tabular}

${ }^{*}$ A01: did the research questions and inclusion criteria for the review include the components of PICO? A02: did the report of the review contain an explicit statement that the review methods were established prior to the conduct of the review and did the report justify any significant deviations from the protocol? A03: did the review authors explain their selection of the study designs for inclusion in the review? A04: did the review authors use a comprehensive literature search strategy? A05: did the review authors perform study selection in duplicate? A06: did the review authors perform data extraction in duplicate? A07: did the review authors provide a list of excluded studies and justify the exclusions? A08: did the review authors describe the included studies in adequate detail? A09: did the review authors use a satisfactory technique for assessing the risk of bias (RoB) in individual studies that were included in the review? A10: did the review authors report on the sources of funding for the studies included in the review? A11: if meta-analysis was performed, did the review authors use appropriate methods for statistical combination of results? A12: if meta-analysis was performed, did the review authors assess the potential impact of RoB in individual studies on the results of the meta-analysis or other evidence synthesis? A13: did the review authors account for RoB in individual studies when interpreting/ discussing the results of the review? A14: did the review authors provide a satisfactory explanation for, and discussion of, any heterogeneity observed in the results of the review? A15: if they performed quantitative synthesis, did the review authors carry out an adequate investigation of publication bias (small study bias) and discuss its likely impact on the results of the review? A16: did the review authors report any potential sources of conflict of interest, including any funding they received for conducting the review?

TABle 4: MAs outcomes about VAS score.

\begin{tabular}{|c|c|c|c|c|c|c|c|c|c|c|c|}
\hline $\begin{array}{l}\text { Study } \\
\text { ID }\end{array}$ & Outcome & $\begin{array}{l}\text { Acute or } \\
\text { preventive } \\
\text { treatment }\end{array}$ & Intervention & Control & $\begin{array}{l}\text { Follow-up } \\
\text { time }\end{array}$ & $\begin{array}{l}\text { RCTs } \\
(n)\end{array}$ & $\mathrm{I} 2$ & Model & MD & $95 \% \mathrm{CI}$ & $P$ value \\
\hline \multirow{3}{*}{$\begin{array}{l}\text { Cui } \\
\text { et al. } \\
{[18]}\end{array}$} & \multirow{3}{*}{ VAS score } & \multirow{3}{*}{ ND } & \multirow[t]{3}{*}{$\begin{array}{l}\text { Acupuncture with or } \\
\text { without electrical } \\
\text { stimulation }\end{array}$} & \multirow{3}{*}{$\begin{array}{l}\text { (1) or } \\
\text { (2) }\end{array}$} & $\begin{array}{l}\text { Immediate- } \\
\text { term (ND) }\end{array}$ & $\begin{array}{c}5 \\
(667)\end{array}$ & $87 \%$ & REM & -0.32 & $\begin{array}{c}{[-1.04,} \\
0.41]\end{array}$ & $<0.0001$ \\
\hline & & & & & $\begin{array}{l}\text { Short-term } \\
\text { (ND) }\end{array}$ & $\begin{array}{c}7 \\
(447)\end{array}$ & $66 \%$ & REM & -1.24 & $\begin{array}{l}{[-1.84} \\
-0.64]\end{array}$ & $<0.0001$ \\
\hline & & & & & $\begin{array}{l}\text { Long-term } \\
\text { (ND) }\end{array}$ & $\begin{array}{c}5 \\
(318) \\
\end{array}$ & $95 \%$ & REM & -1.77 & $\begin{array}{l}{[-2.76,} \\
-0.78]\end{array}$ & 0.0005 \\
\hline \multirow{4}{*}{$\begin{array}{l}\mathrm{Pu} \\
\text { et al. } \\
{[25]}\end{array}$} & \multirow[b]{2}{*}{ VAS score } & \multirow{4}{*}{$\begin{array}{c}\text { Acute } \\
\text { treatment }\end{array}$} & \multirow{4}{*}{ Acupuncture } & \multirow{4}{*}{ (1) } & $2 \mathrm{~h}$ & $\begin{array}{c}4 \\
(699)\end{array}$ & $67 \%$ & REM & -0.38 & $\begin{array}{c}{[-0.83,} \\
0.07]\end{array}$ & 0.10 \\
\hline & & & & & $4 \mathrm{~h}$ & $\begin{array}{c}4 \\
(699)\end{array}$ & $70 \%$ & REM & -0.42 & $\begin{array}{c}{[-0.96} \\
0.12]\end{array}$ & 0.12 \\
\hline & \multirow{2}{*}{$\begin{array}{l}\text { VAS score } \\
\text { reduction }\end{array}$} & & & & $2 \mathrm{~h}$ & $\begin{array}{c}3 \\
(579)\end{array}$ & $0 \%$ & FEM & 0.36 & $\begin{array}{l}{[0.08} \\
0.65]\end{array}$ & 0.01 \\
\hline & & & & & $4 \mathrm{~h}$ & $\begin{array}{c}3 \\
(579)\end{array}$ & $0 \%$ & FEM & 0.49 & $\begin{array}{l}{[0.14,} \\
0.84]\end{array}$ & 0.01 \\
\hline
\end{tabular}

${ }^{*}$ ND: not defined; (1): sham acupuncture; (2): drug treatment.

acupuncture has the advantage in pain improvement of VAS score, headache days/frequency, analgesic use and efficacy of response rate, and effective rate according to the present evidence. However, most SR/MAs included did not conclude firmly because of the small size or low methodological quality of the included trials and subjectively evaluated outcome of included trials, and some SR/MAs also stated that high-quality acupuncture for migraine RCT was still needed to confirm the main findings further. Based on previous evidence we reviewed, we supposed acupuncture might be a kind of available treatment for migraine in preventive or acute treatment, but in consideration of low 


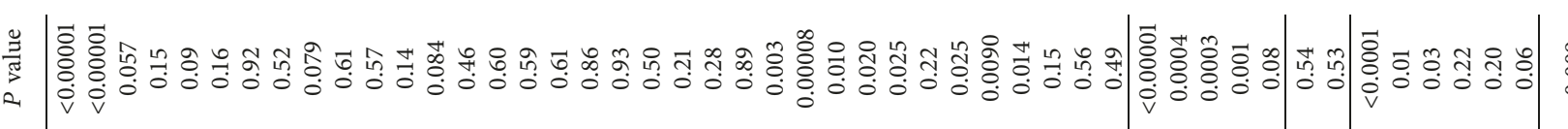

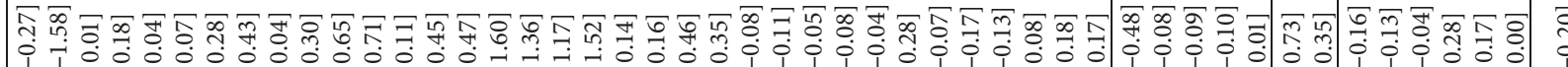

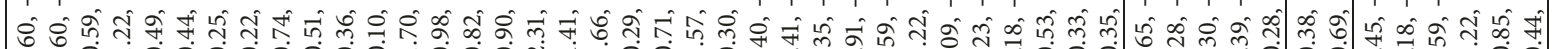

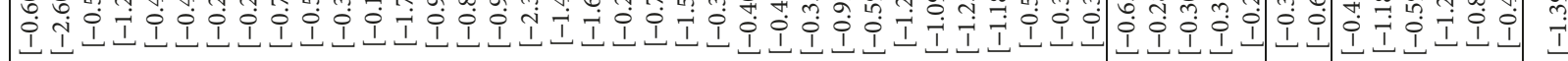

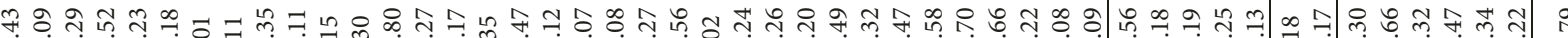

跔

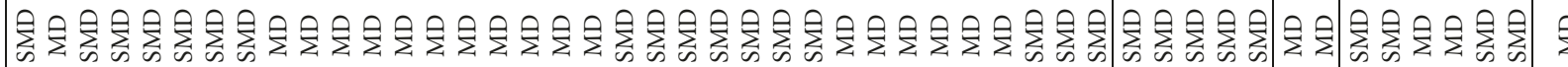

㺃

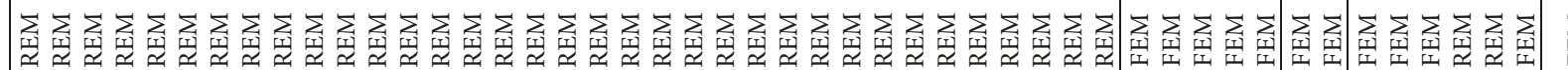

$\simeq$

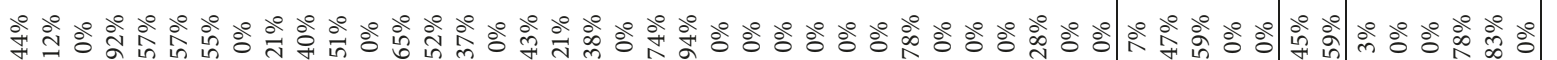

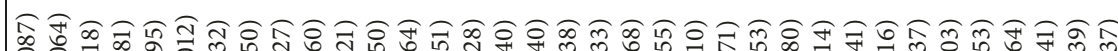

我

ㅎํㄷำ 


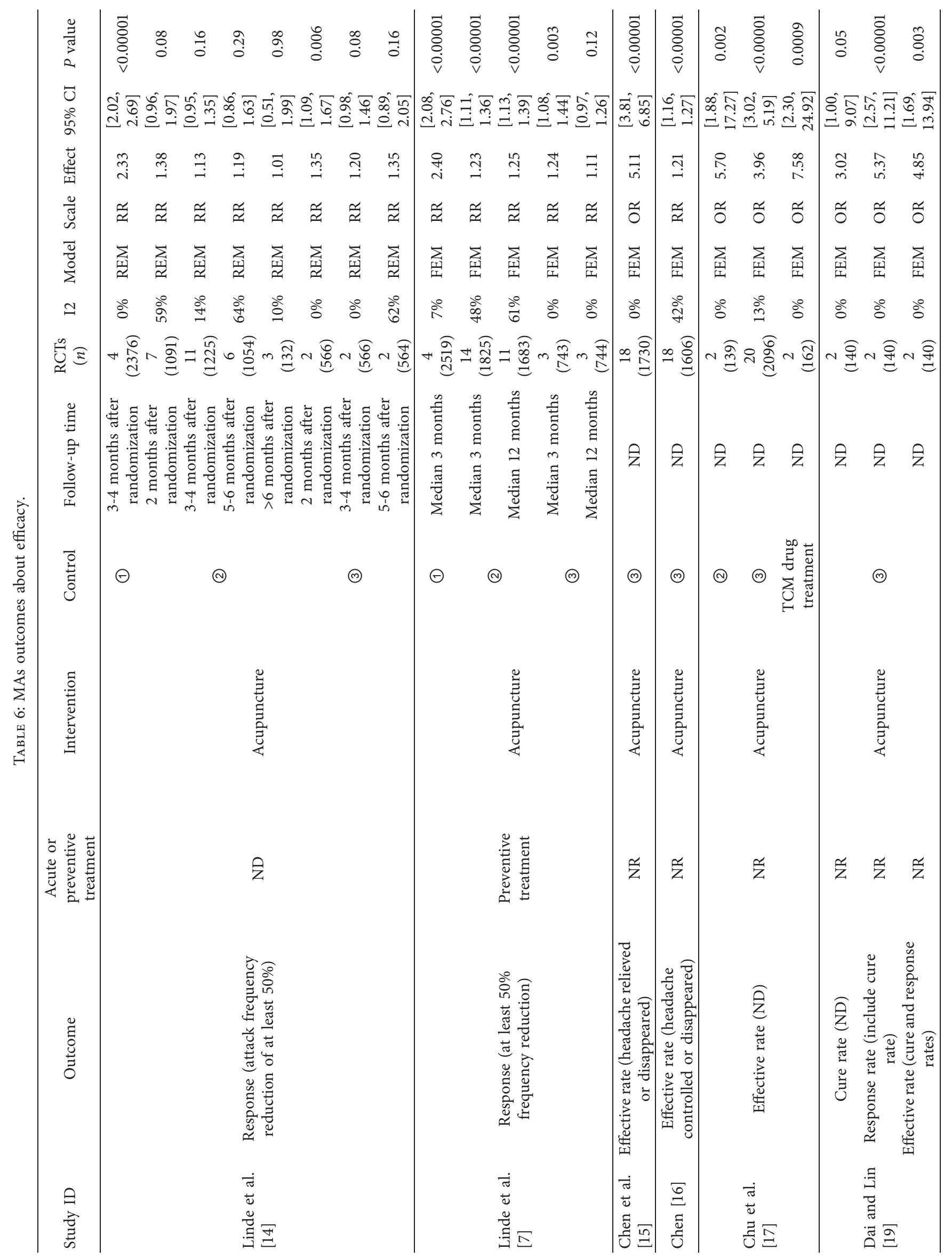




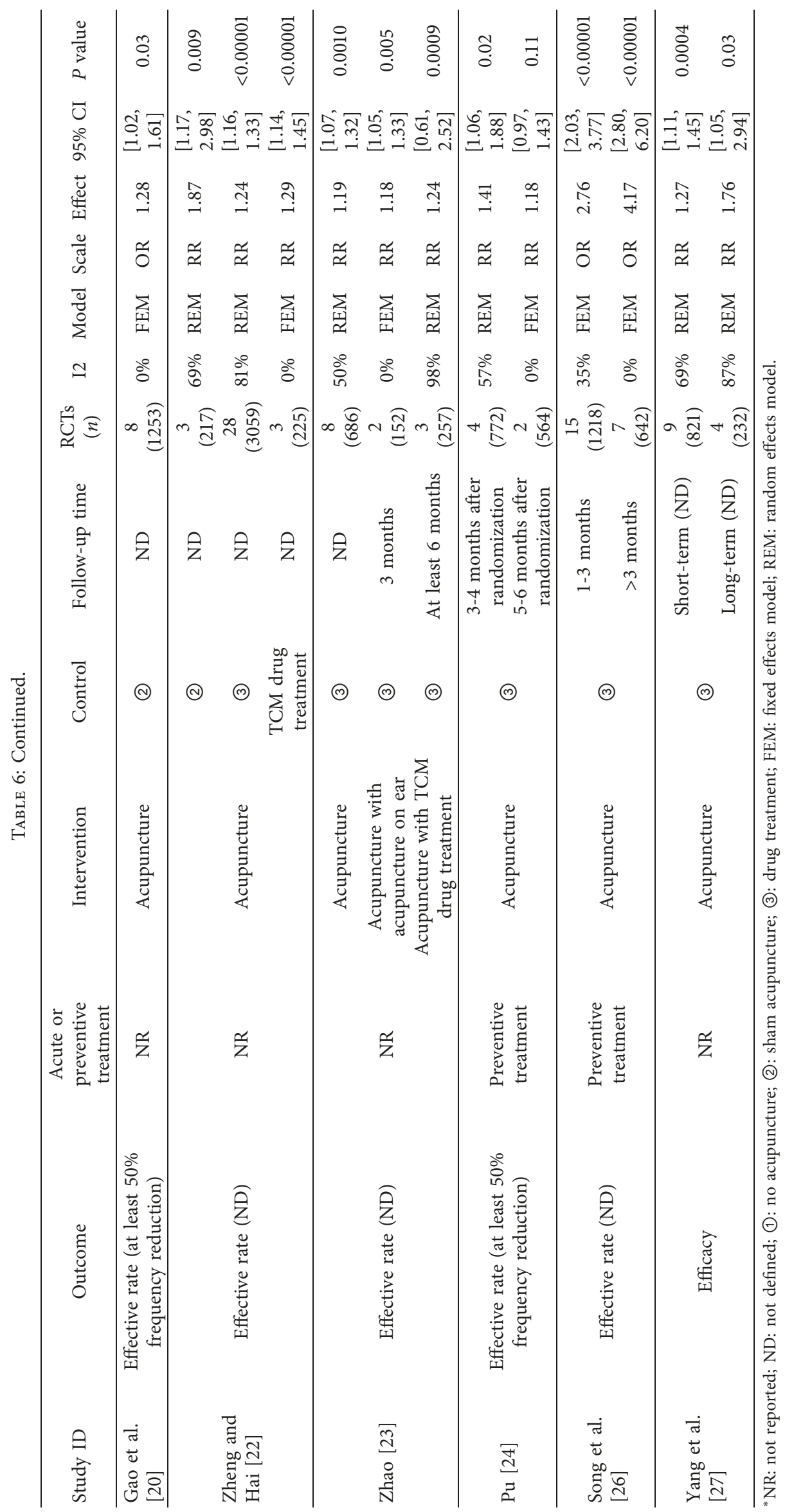




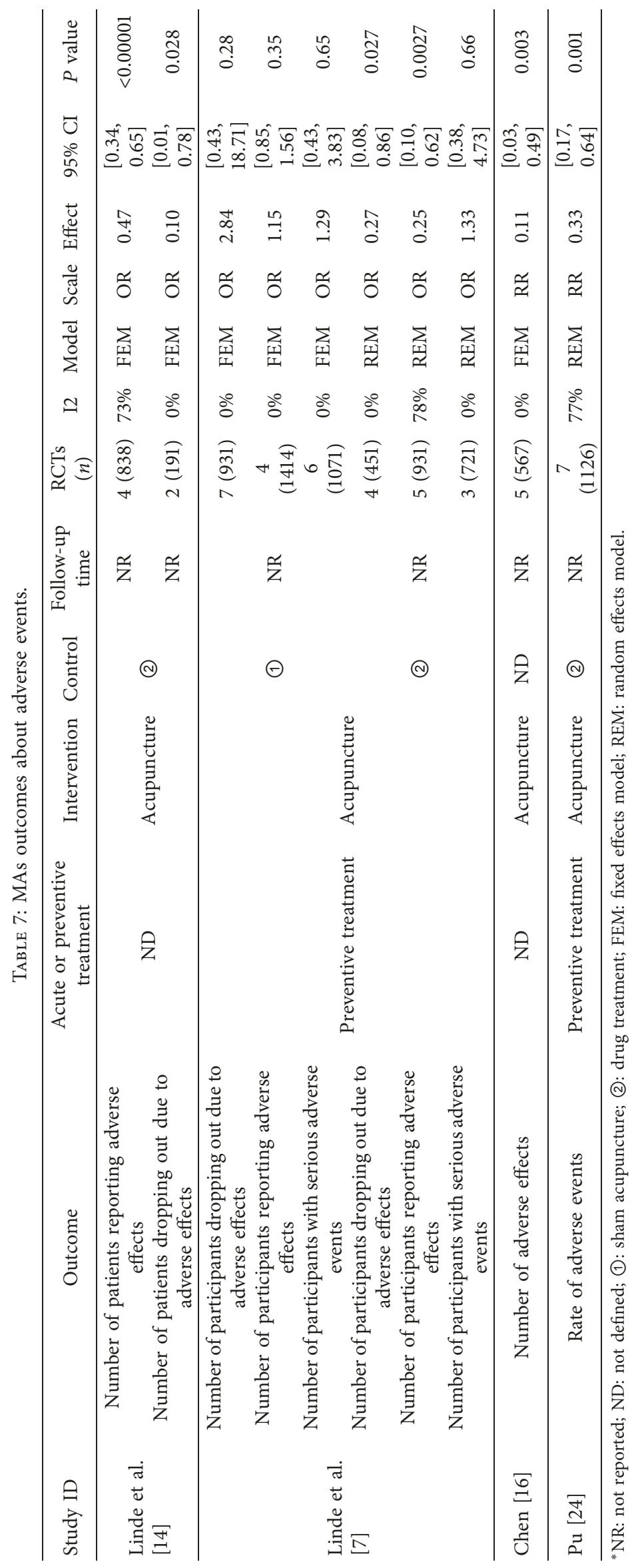


methodological quality of present SR/MAs or RCTs of acupuncture for migraine, we need more high-quality evidence to demonstrate the effect of acupuncture for migraine.

5.2. Designs of Present SR/MAs. There was some advice for later researchers to carry out high-quality SR/MAs or RCTs of acupuncture for migraine, which was summarized according to designs of present SR/MAs.

5.3. Participants. Most studies included did not mention whether the patients they included were with acute attacks of migraines or not, which reduce the significance of most included SR/MAs in revealing the suitable timings of applying acupuncture for migraines. Therefore, later researchers can get more meaningful results with a more clear statement of patients' conditions like whether they are with aura or attacks.

5.4. Control Group. The control interventions included in SR/MAs were blank control, sham acupuncture, and drug treatment. In addition, one of the heterogeneity sources may be regarding different types of drug treatment as 1 kind of subgroup. As a result, later researchers should provide a clear comparison of intervention vs. control in their studies, which can not only improve the robustness of results but also offer suggestions of combined therapy including acupuncture in clinical practice.

5.5. Outcome. The most common outcomes were about an effective rate. And results of SR/MAs in most outcomes were contradictory, which means we inferred that the efficacy is evaluated by both patient's own pain feeling and the estimate of different doctors; therefore, a certain degree of subjectivity in evaluation might appear, hence the difference in medical sets, communicating skills of doctors or other factors in each SR/MA, which can lead to bias in the conclusions of SR/ MAs. We consider that SR/MAs and RCTs of acupuncture for migraine have problems with outcome indexes, for instance, outcomes were difficult to be evaluated stably and were also not international recognized like other clinical studies of other TCM treatments [31]. So, later researchers can also increase the reliability of their studies by regarding international recognized outcomes as outcomes in their studies.

5.6. Methodological Quality and Evidence Certainty of Systematic Reviews Included. Researchers of included SR/MAs generally concluded that the reliability of their own SR/MAs evidence was low, more high-quality, large sample of acupuncture treating migraine RCT trials were needed, and the low quality of acupuncture treating migraine may affect the reliability of the evidence of SR/MAs in acupuncture for migraine. In addition, this study suggests that some researchers of SR/MAs did not synthesize data of different follow-up time and whether were grouped with acute attacks or not, respectively, which may also affect the heterogeneity of results provided by SR/MAs and reduce the certainty of their studies. And there were only 4 studies using GRADE to review the certainty of the evidence, whereas most metaanalysis outcomes were low or very low certainty. As assessment of AMSTAR-2, SR/MAs in acupuncture treating migraine need to be improved in methodology and reporting quality as these points below: lack of research protocol statement and registration may affect the transparency of the research results, lack of showing and explaining the reason of excluding studies may leave some information missing, lack of revealing funding sources of included studies may make researchers or readers ignore potential benefits and conflicts, and lack of statement of self-interest relationship may make readers ignore factors affecting objectivity and reliability of SR/MAs.

The core reason of low methodological quality of SR/ MAs may be that a large portion of researchers of SR/MAs in acupuncture for migraine may not receive standard evidence-based medical education; therefore, we should strengthen and improve the work of evidence-based medicine education in higher education and continuing education of TCM, especially with the selection of suitable outcomes.

5.7. Strength and Limitations. The strengths of this study were as follows: (1) both English and Chinese studies were included to promise included studies widely and (2) each MAs outcome was showed in structured tables which can help readers realize or review interesting outcomes easily. The limitations of this study were as follows: (1) SR/MAs included were all low quality, which may reduce the confidence of the results, and (2) the conflicts of interest of SR/ MAs included were not analyzed, which may induce to miss some information.

\section{Conclusions}

From this study, we found that acupuncture has the advantage for acute and preventive treatment of migraine in pain improvement and safety, but the quality of SR/MAs of acupuncture for migraine still needs to be improved.

\section{Conflicts of Interest}

None of the authors has any financial conflicts of interest to disclose.

\section{Authors' Contributions}

Xia-tian Zhang, Chen Zhao, and Xin-yi Li contributed to designing the study, analyzing data, and drafting the manuscript. Ye-yin $\mathrm{Hu}$ performed screening data, searching studies, and managing data. Yi-yi Lin contributed to drawing and checking data. He-qing Chen performed screening data and drawing data; Zhao-feng Shi and Xiao-yu Zhnag contributed to drawing data. Hong-cai Shang drafted the manuscript. and Gui-hua Tian designed the study and is responsible for this study. Xia-tian Zhang, Chen Zhao, and Xin-yi Li contributed equally to this work. 


\section{Acknowledgments}

The National Natural Science Fund (no. 81674050).

\section{References}

[1] Headache Classification Committee of the International Headache Society (IHS), "The international classification of headache disorders, 3rd edition (beta version)," Cephalalgia, vol. 33, no. 9, pp. 629-808, 2013.

[2] L. J. Stovner, E. Nichols, T. J. Steiner et al., "Global, regional, and national burden of migraine and tension-type headache, 1990-2016: a systematic analysis for the Global Burden of Disease Study 2016," The Lancet Neurology, vol. 17, no. 11, pp. 954-976, 2018.

[3] S. Yu, R. Liu, G. Zhao et al., "The prevalence and burden of primary headaches in China: a population-based door-todoor survey," Headache: The Journal of Head and Face Pain, vol. 52, no. 4, pp. 582-591, 2012.

[4] M. J. Marmura, S. D. Silberstein, and T. J. Schwedt, "The acute treatment of migraine in adults: the American headache society evidence assessment of migraine pharmacotherapies," Headache: The Journal of Head and Face Pain, vol. 55, no. 1, pp. 3-20, 2015.

[5] D. W. Dodick, "Migraine," The Lancet, vol. 391, no. 10127, pp. 1315-1330, 2018.

[6] B. M. Berman, H. M. Langevin, C. M. Witt, and R. Dubner, "Acupuncture for chronic low back pain," New England Journal of Medicine, vol. 363, no. 5, pp. 454-461, 2010.

[7] K. Linde, G. Allais, B. Brinkhaus et al., "Acupuncture for the prevention of episodic migraine," Cochrane Database of Systematic Reviews, vol. 6, Article ID CD001218, 2016.

[8] G. Zhong and W. Li, "Effects of acupuncture on 5-hydroxytryptamine $1 \mathrm{~F}$ and inducible nitricoxide synthase gene expression in the brain of migraine rats," Chinese Journal of Tissue Engineering Research, vol. 11, no. 29, pp. 5761-5764, 2007.

[9] J. Gurevitch, J. Koricheva, S. Nakagawa, and G. Stewart, "Meta-analysis and the science of research synthesis," Nature, vol. 555, no. 7695, pp. 175-182, 2018.

[10] J. Higgins and S. Green, "Cochrane handbook for systematic reviews of interventions. The Cochrane collaboration," Naunyn-Schmiedebergs Archiv für experimentelle Pathologie und Pharmakologie, vol. 5, no. 2, p. S38, 2011.

[11] R. H. Dworkin, D. C. Turk, J. T. Farrar et al., "Core outcome measures for chronic pain clinical trials: IMMPACT recommendations," Pain, vol. 113, no. 1-2, pp. 9-19, 2005.

[12] B. J. Shea, B. C. Reeves, G. Wells et al., "Amstar 2: a critical appraisal tool for systematic reviews that include randomised or non-randomised studies of healthcare interventions, or both," BMJ, vol. 358, p. j4008, 2017.

[13] H. J. Schünemann, A. D. Oxman, J. Brozek et al., "Grading quality of evidence and strength of recommendations for diagnostic tests and strategies," $B M J$, vol. 336, no. 7653, pp. 1106-1110, 2008.

[14] K. Linde, G. Allais, B. Brinkhaus, E. Manheimer, A. Vickers, and A. R. White, "Acupuncture for migraine prophylaxis," Cochrane Database of Systematic Reviews, vol. 1, p. CD001218, 2009.

[15] L. Chen, X. He, H. Xu, Z. Zhou, C. Liu, and Q. Yang, "Metaanalysis of randomized controlled clinical trials' literature of acupuncture treating migraine," Hunan Jorunal of Traditional Chiese Medicine, vol. 2, pp. 125-129, 2018.
[16] W. Chen, Systematic Reviews for Short-Term Effects of Acupuncture in Treating Migraine, Chengdu University of Traditional Chinese Medicine, Chengdu, China, 2014.

[17] X. Chu, R. Du, H. Cui et al., "Meta-analysis of acupuncture treating migraine in recent 10 years," Continuing Medical Education, vol. 4, pp. 157-158, 2018.

[18] Q. Cui, Q. He, J. Fang, X. Shao, and C. Zhou, "Meta-analysis of time-effect evaluation of acupuncture treatment for migraine," Shanghai Journal of Acupuncture and Moxibustion, vol. 4, pp. 466-473, 2018.

[19] X. Dai and C. Lin, "A meta-analysis of treating migraine by acupuncture," Clinical Journal of Chinese Medicine, vol. 7, no. 35 , pp. 1-3, 2015.

[20] X. Gao, B. Wang, Y. Song, Q. Hong, Z. Rong, and H. Wang, "Acupuncture treatment of migraine: a systematic review and meta-analysis," Chinese Journal of Clinical Pharmacology and Therapeutics, vol. 5, pp. 530-537, 2011.

[21] Y. Yang, Q. Que, X. Ye, and G. h. Zheng, "Verum versus sham manual acupuncture for migraine: a systematic review of randomised controlled trials," Acupuncture in Medicine, vol. 34, no. 2, pp. 76-83, 2016.

[22] S. Zheng and C. Hai, "Acupuncture for migraine: a meta analysis Chinese," Journal of Information on Traditional Chinese Medicine, vol. 6, pp. 20-23, 2012.

[23] Y. Zhao, Data Mining and Systematic Review of Modern Acupuncture Treatment for Menstrual Migraine Master, Chengdu University of Traditional Chinese Medicine, Chengdu, China, 2016.

[24] S. Pu, Effectiveness and Safety of Acupuncture versus Drug Treatment for Migraine Prophylaxis: A Meta-Analysis, Chongqing Medical University, Chengdu, China, 2016.

[25] S. Pu, G. Tan, D. Yanwang, J. Chen, and L. Jiang, “Analgesic effect of acupuncture during migraine acute attack period: a meta-analysis," Chongqing Medicine, vol. 45, no. 10, pp. 1353-1356, 2016.

[26] Q. Song, S. Zhao, L. Li, Y. Shen, and S. Wang, "Meta-analysis on prevention comparison of acupuncture with western medicine for migraine," Liaoning Journal of Traditional Chinese Medicine, vol. 4, pp. 821-826, 2016.

[27] J. Yang, Y. Shen, and S. Wang, "Systematic review on efficacy for migraine treatment by acupuncture and flunarizine," Modernization of Traditional Chinese Medicine and Materia Medica-World Science and Technology, vol. 7, pp. 1608-1613, 2014.

[28] J. P. T. Higgins, D. G. Altman, P. C. Gotzsche et al., "The Cochrane Collaboration's tool for assessing risk of bias in randomised trials," BMJ, vol. 343, p. d5928, 2011.

[29] H. D. Clark, G. A. Wells, C. Huët et al., "Assessing the quality of randomized trials," Controlled Clinical Trials, vol. 20, no. 5, pp. 448-452, 1999.

[30] Traditional Chinese Medicine, Criteria for the Diagnosis and Therapeutic Effect of Diseases and Symptoms in Traditional Chinese Medicine, Bejing: Chinese Medicine Publishing House, Bejing, China, 2012.

[31] C. Yu, Y. Sun, L. He, and J. Fan, "Establishment of core outcome sets in the trials of traditional Chinese medicine," China Journal of Traditional Chinese Medicine and Pharmacy, vol. 7, pp. 2526-2529, 2016. 


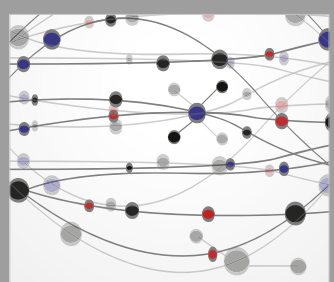

The Scientific World Journal
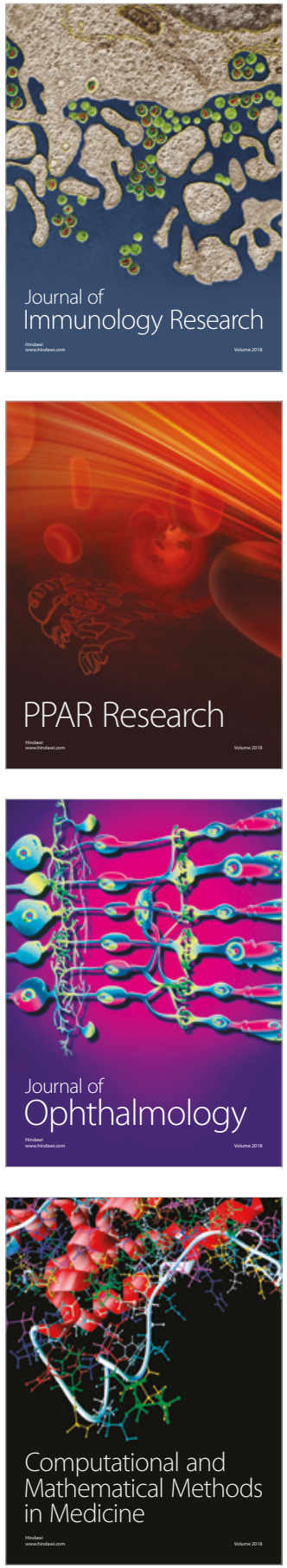

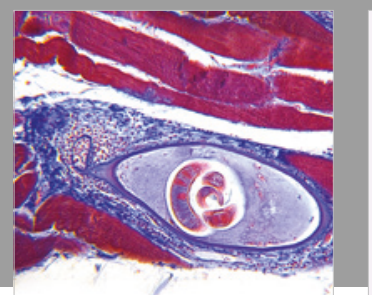

Gastroenterology Research and Practice

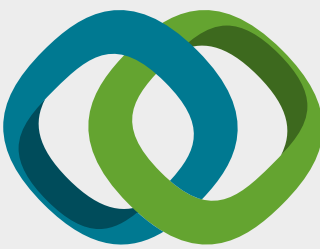

\section{Hindawi}

Submit your manuscripts at

www.hindawi.com
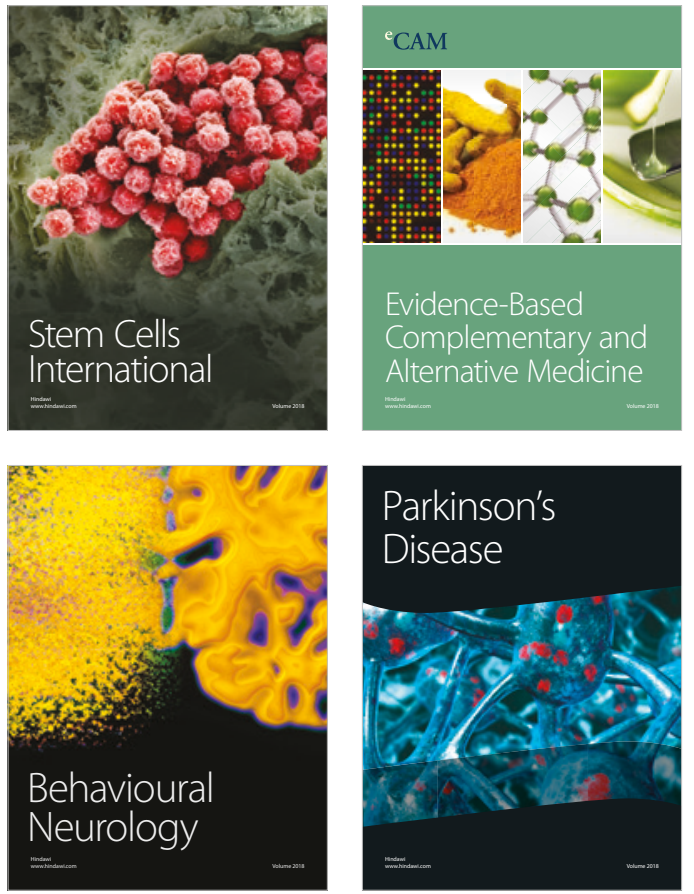

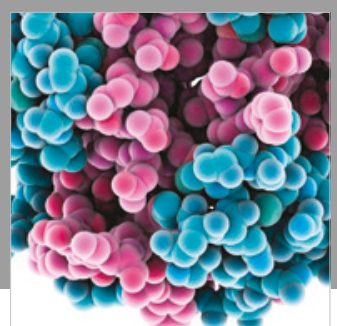

ournal of

Diabetes Research

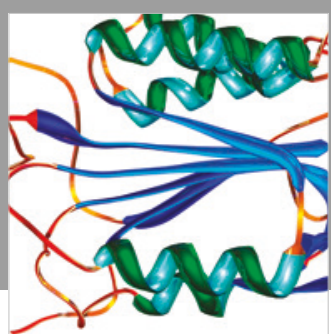

Disease Markers
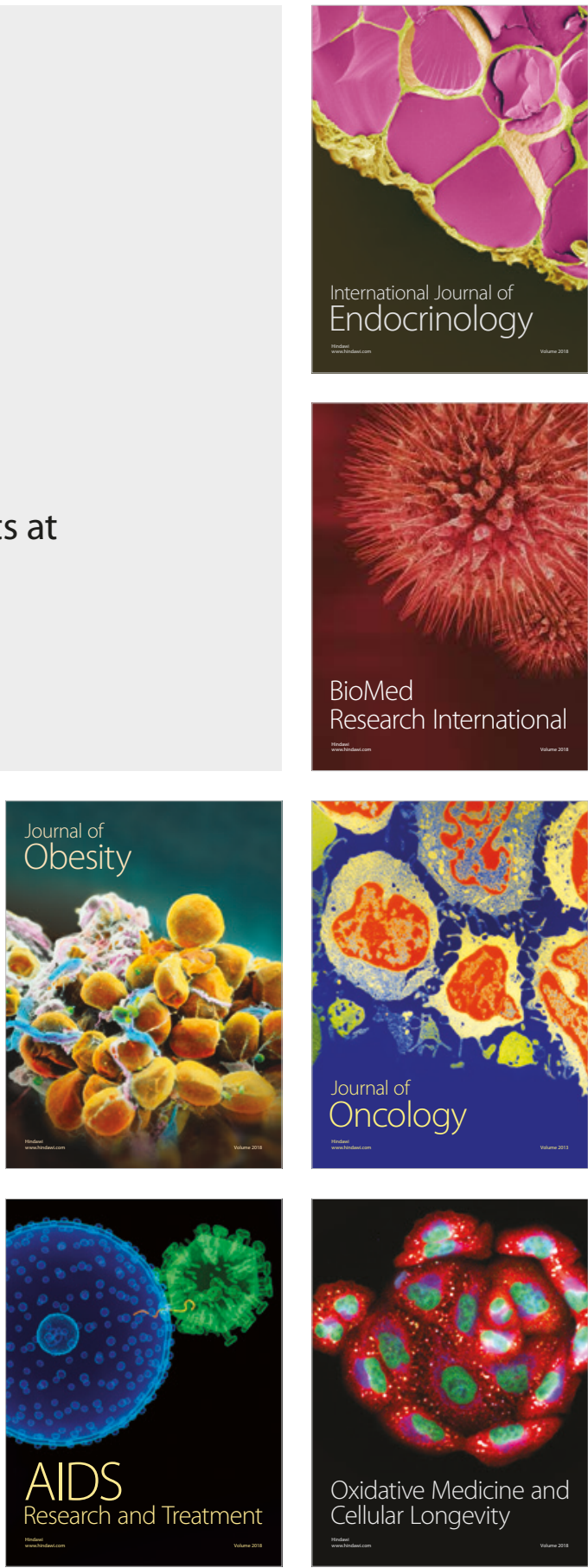